\title{
Knowledge and practices related to sexually transmitted infections among women of reproductive age living in Katanga slum, Kampala, Uganda.
}

\author{
Faith Nawagi ${ }^{1}$, Arthur Mpimbaza ${ }^{2}$, John Mukisa ${ }^{3}$, Patrick Serwadda ${ }^{3}$, Samuel Kyalema ${ }^{3}$, Daniel Kizza ${ }^{4}$
}

1. Department of Nursing, Makerere University-College of Health Sciences, P. O. Box 7072 Kampala, Uganda.

2. Child Health Development Centre, Makerere University-College of Health sciences, P. O. Box 7072, Kampala, Uganda

3. School of Medicine, Makerere University-College of Health sciences. P. O. Box 7072 Kampala, Uganda.

4. School of Health Sciences, Makerere University-College of Health sciences, P. O. Box 7072, Kampala, Uganda

\begin{abstract}
Background: Sexually transmitted infections (STIs) still stand as one of the commonest health problems affecting women of reproductive age. The knowledge and practices of STIs, among susceptible populations such as women of reproductive age, living in slums like Katanga in Kampala Uganda need to be established.

Methods: This was a cross- sectional study with 339 participants in Katanga slum. Data was collected using an interviewer administered questionnaire, entered and analysed using SPSS version 17.0. Data was summarized using frequencies for categorical data and medians for continuous data.

Results: Majority of the participants $(71.9 \%)$ were $\geq 25$ years with a mean age of $28.0(\mathrm{SD} \pm 7.0)$ years. The commonest symptoms known to the participants were genital itching $(60 \%)$ and genital rash (14.5\%). Most mentioned multiple partners $(63.7 \%)$ and unprotected sex $(50.7 \%)$ as predisposing factors to STIs. Knowledge on methods of prevention was high $(92.3 \%)$ however, $18.8 \%$ were found positive for STIs using the syndromic approach and $82 \%$ mentioned having suffered from STIs in the past 6 months more than once.

Conclusion: Most participants did not know about the systemic effects of STIs to their health and didnot follow the appropriate behavior patterns despite being knowledgeable about the various methods of prevention of STIs.

Keywords: STI-Sexually transmitted infections, Katanga slum.

DOI: http://dx.doi.org/10.4314/ahs.v16i1.15

Cite as: Nawagi F, Mpimbaza A, Mukisa J, Serwadda P, Kyalema S, Kizza D. Knowledge and practices related to sexually transmitted infections among women of reproductive age living in Katanga slum, Kampala, Uganda. Afri Health Sci. 2016;16(1): 116-122. http:/ / dx.doi. org/10.4314/ahs.v16i1.15
\end{abstract}

\section{Introduction}

Sexually Transmitted Infections (STIs) are a group of diseases that are spread through sexual intercourse and mainly affect the human reproductive tract. STIs are a common public health problem worldwide, with developing countries accounting for majority of cases. In 2008, the World Health Organization estimated that up to 499 million cases of curable STIs occurred that year, majority occurring in low income countries ${ }^{1}$. In Ugan$\mathrm{da}$, the prevalence of STI among sexually active adoles-

\section{Corresponding author: \\ Faith Nawagi, \\ Department of Nursing, \\ Makerere University, College of Health Sciences, \\ P O Box 7072, Kampala, Uganda.}

Tel: 256775491447.

Email:fnawagi@gmail.com cents attending a health care clinic was found to be as high as $20.6 \%$ among females and $13.2 \%$ among males 2 . More recently, the national prevalence of syphilis was found to be $2 \%{ }^{3}$.

Infectious agents that cause STI can be classified as either viral, bacterial, protozoal or funga ${ }^{4-5}$. STIs can result in serious long term complications including pregnancy complications, fetal and neonatal demise, cancer, infertility, sexual dysfunction, and enhanced HIV transmission $^{6-8}$. In addition, STIs have been shown to have serious physical, psychological and social consequence, negatively impacting on social and economic well being of affected persons ${ }^{9-10}$. Populations at risk include adolescents, sex workers and their clients, men who have sex with men, prisoners, truck drivers, fishing communities. Additionally, compared to men, women are at an increased risk of acquiring STI, a fact attributed to gender inequity and biological predisposition ${ }^{11}$. 
Despite efforts to control the spread of STIs, reports indicate an upward surge in the incidence of new cas$\mathrm{es}^{1}$. This situation is explained by a number of factors including, growing resistance to the common treatment regimens, lack of prioritization of management of STIs with the exception of HIV, STI surveillance, prevention and treatment programmes are generally under staffed and poorly resourced undermining the quality of services rendered by most National programs ${ }^{14-15}$.

In-addition to biomedical primary prevention strategies such as vaccines, circumcisions, behavioral interventions are a major component of Uganda's strategy towards STI control in the country ${ }^{16}$. Persistent high levels of STI prevalence suggest that uptake of these interventions remains sub-optimal. To inform the design of effective behavioural preventive strategies, we conducted a cross sectional study to determine knowledge and practices related to sexually transmitted infections (STIs) among a high risk population, comprised of women of reproductive age living in Katanga slum, Kampala Uganda.

\section{Materials and methods \\ Study area}

This study was conducted in Katanga, an urban slum located in Kampala, the capital city of Uganda. Katanga is one of the biggest slums in Kampala, with a population of over 20,000 people, of which more than $50 \%$ are children of very low socio-economic status. The slum has a large number of sex workers, vendors, and brewers of local alcoholic drinks.

\section{Study design}

This was a cross sectional study design.

\section{Sample size}

Our sample size estimate was determined based on the results of a survey conducted in Kampala, that found the prevalence of STIs among women prostitutes to be $33 \%{ }^{12}$. Using Kish, Leslie formula we required a sample size of 335 women to detect an STI prevalence of $33 \%$ at a level of significance equal to or less than 0.05 and with power 0.80 .

\section{Eligibility criteria}

Women aged 18 to 45 years, and who were permanent residents of the Katanga slum in Kampala Uganda, were eligible for inclusion in the study. Critically ill women were excluded from the study. Eligible partic- ipants who provided written informed consent were enrolled into the study.

\section{Sampling technique and selection of households}

Households with eligible participants where stratified into four zones, and consecutively approached within each zone. With the help of local leaders, one of 4 interview teams approached households, where eligible women would then be identified, screened and enrolled. If a woman of reproductive age was not present at home at the time of being approached, that household was excluded.

\section{Data collection}

A pre-tested interviewer administered questionnaire designed based on the WHO simplified tool for diagnosis and treatment of STIs was used to collect information from enrolled study participants ${ }^{13}$. Information collected included; demographic characteristics, socio-economic status, education level, knowledge of types, causes and symptoms of STIs, and prevention methods. Diagnosis of STIs was based on a syndrome approach applied to information captured using the questionnaire. Participants were asked to report only one symptom that they considered to be most associated with an STI.

\section{Data management}

All forms for data collection were serialized for proper data management and recording. Data collected was checked for completeness and double entered with EPI data 3.1.

Qualitative data was coded, transcribed and double checked respectively. All data entered was analyzed using SPSS 17.0. Descriptive statistics such as mean, median and standard deviations (SD) were done for continuous variables. Frequency distribution and percentages were done for categorical variables. The analyzed data was then presented in the form of tables, figures and texts.

\section{Ethical consideration and confidentiality issues}

Ethical clearance to conduct the study was sought from the Makerere University School of Biomedical Sciences Research and Ethics Committee. Approval was also sought from the Uganda National council of science and technology (UNSCT). Questionnaires were anonymised, and records kept confidentially. Only study investigators had access to patient information. This study was conducted in accordance with guidelines of conduct of research as stipulated by the WHA. Writ- 
ten consent was sought from all participants before enrollment into the study and privacy was ensured during data collection.

\section{Limitation of the study}

The study was dependent on the number of people who were willing to consent and take part in the study and the trustworthiness of the participants for the information given.

\section{Quality control}

A pilot study was conducted among 10 resident women of reproductive age in Kamokya, a suburb slum prior to the rollout of the main study. During this period knowledge about STIs was assessed. This was to aid in development of a good data collection tool, observe reactions of the respondents to the research procedure such that adjustments are made where necessary.

\section{Results}

Social demographic characteristics of participants Between September and November 2013, we approached 339 women of reproductive age living in Katanga slum, and all agreed to participate in the study. Majority of study participants $(71.9 \%)$ were aged 25 years and above with a mean age of 28.0 ( $\mathrm{SD} \pm 7.0$ ) years (Table 1). The youngest study participant was 18 years of age while the oldest was 48 years. Majority of participants were married (58\%) and nearly half had attained secondary level of education (49\%). More than a quarter $112(33 \%)$ of the participants had four or more children with a mean number of $3.0(\mathrm{SD} \pm 2)$ children.

Table 1: Social demographic characteristics of study participants

\begin{tabular}{ll}
\hline Variable & $\mathbf{N}=\mathbf{3 3 9}$ \\
\hline Age in years, mean (+/-SD) & $28(+/-7)$ \\
$<25$ & $95(28.1 \%)$ \\
$=25$ & $244(71.9 \%)$ \\
Marital status, $\mathbf{n}$ (\%) & \\
Single & $143(42.2 \%)$ \\
Married & $195(57.5 \%)$ \\
Divorced & $1(0.3 \%)$ \\
Number of children, $\mathbf{n}$ (\%) & \\
$=3$ & $22767 \%)$ \\
$=4$ & $112(33 \%)$ \\
Level of education, $\mathbf{n}(\%)$ & \\
Primary & $136(40.1 \%)$ \\
Secondary education (A-level) & $21(6.2 \%)$ \\
Secondary education (0-level) & $166(49 \%)$ \\
Graduate & $16(4.7 \%)$ \\
\hline
\end{tabular}

Knowledge about STIs among study participants Most participants were aware of types of STIs, with $88 \%$ of respondents mentioning at least one of the following STIs, syphilis, candida, HIV, and gonorrhea. The commonest symptoms of STIs reported were gen- ital itching (60\%) and genital rash (14.5\%; Table 2). In terms of how STIs are transmitted, $63.7 \%$ and 50.7 $\%$ of participants mentioned multiple sexual partners and unprotected sex as predisposing factors for STIs respectively. However, only $8.6 \%$ knew that STIs were caused by micro organisms like bacteria and viruses. 


\begin{tabular}{ll}
\hline Symptoms, n (\%) & $\mathbf{N}=\mathbf{3 3 9}$ \\
\hline Genital itching & $202(60 \%)$ \\
Genital ulcer & $13(3.8 \%)$ \\
Thick smelly discharge & $13(3.8 \%)$ \\
Genital rash & $49(14.5 \%)$ \\
Pain on urination & $16(4.7 \%)$ \\
Loss of weight & $20(5.9 \%)$ \\
Abdominal pain & $10(2.9 \%)$ \\
Painful sex & $1(0.3 \%)$ \\
Fever & $7(2.1 \%)$ \\
Not known & $8(2.4 \%)$
\end{tabular}

Only $169(51 \%)$ could identify specific types of STIs based on signs and symptoms with $71.9 \%$ knowing that they are treatable and curable. Half of the participants $169(50 \%)$ lacked knowledge on the complications of
STIs. Knowledge on methods of prevention of spread of STIs were high (92.3\%), with condom use (49\%) being the most frequently reported methods of prevention of spread of STIs (Table 3).

Table 3: Distribution of participants by knowledge on methods of STI prevention.

\begin{tabular}{lc}
\hline Prevention, n (\%) & $\mathbf{N = 3 3 9}$ \\
\hline Regular checkups at hospital" & $8(2.4 \%)$ \\
Being faithful by having one sexual partner & $89(26.3 \%)$ \\
Abstinence & $22(6.5 \%)$ \\
Condom use & $166(49 \%)$ \\
Taking prophylaxis drugs" & $1(0.3 \%)$ \\
Using clean bathroom and toilets & $10(2.9 \%)$ \\
Proper hygiene by bathing" & $15(4.4 \%)$ \\
not sharing sharp instruments" & $2(0.6 \%)$ \\
Don't know" & $26(7.7 \%)$ \\
\hline
\end{tabular}


Using the syndromic approach, $18.8 \%$ of respondents were found positive for STIs, and $82 \%$ mentioned having suffered from STIs in the past 6 months prior to the date of interview. Among those who were found positive for STIs using the syndromic approach, 76.6\% accessed formal health care services at a hospital or clinic, $7.8 \%$ used herbal medicine while $15.6 \%$ had not yet sought any medical attention. The main source of information about STIs was from Mulago National referral hospital, which neighbors the slum (Table 4)

\section{Table 4: Distribution of participants by the sources of information on STIs.}

\begin{tabular}{lc}
\hline $\begin{array}{l}\text { Sources of information,n } \\
\text { (\%) }\end{array}$ & $\mathbf{N}=\mathbf{3 3 9}$ \\
\hline Hospital & $137(40.4 \%)$ \\
Media & $81(23.9 \%)$ \\
Friend & $91(26.8 \%)$ \\
School & $25(7.5 \%)$ \\
Don't know & $5(1.4 \%)$ \\
\hline
\end{tabular}

\section{Discussion}

We carried out a cross sectional study to determine the knowledge, practices and prevalence of STIs in a high risk population of women of reproductive age living in an urban slum in Uganda. Consistent with previous studies, we found a high prevalence of STIs in this population. Levels of knowledge in terms of methods of transmission of STIs were high, however levels of knowledge in terms of methods of prevention of spread of STI were modest, particularly so with respect to the current strategies being promoted by the Uganda National health programmes. The high prevalence of STIs in this high risk population amidst relatively high levels of knowledge of STI highlights the challenges of translating knowledge into practice, particularly in vulnerable populations, like the one we studied.

Interestingly, we found that many respondents in this population were not aware of the common predisposing factors of STIs in Uganda with nearly all mentioning at least one of the common predisposing factors. These findings are in contrast to those of a study conducted in a similar set up in Bangladesh which found only $31 \%$ of the participants were aware of types of STIs ${ }^{17}$. Difference in findings may be attributed to the higher level of education noted in our study population. In addition, the close proximity of the slum to a National Referral hospital may have positively impacted on knowledge gain in our study population.

The most frequently reported symptoms included genital itching, burning and genital rash which may be reflective of the commonest form of STIs in Ugan- da including candidiasis, trichomas vaginalis, gonoccal infection and chlamydia trachomatis.

A small proportion of participants did not know anything regarding predisposing factors for STIs with most mentioning multiple partners $(63.7 \%)$ and unprotected sex $(50.7 \%)$ as the major predisposing factors. Despite being the most frequently reported forms of prevention of spread of STIs; these levels are relatively low considering that condom use and avoidance of multiple sexual partners are key messages in Uganda's strategy to control spread of STIs and HIV ${ }^{16}$. Indeed, these findings suggest that campaign effort by the health sector through government and non-governmental organizations has to an extent played an impactful role to sensitize the population on STIs through mass media and health talks at the various health centers. However, despite resulting in increased levels of awareness, desired target levels are yet to be realized ${ }^{16}$.

There was a significant knowledge gap with close to half of the participants (40\%) having no clue on the effects and complications of STIs on their health and body, and only $8.6 \%$ knowing that STIs were caused by microorganisms like bacteria and viruses. This simply means that much of the health education talks address signs and symptoms, predisposing factors and prevention with less emphasis being put on the negative impact of STIs on one's health and body system and causative microorganisms.

Although knowledge on the various methods of prevention as shown in Table 3 was high $(92.3 \%)$, it was not followed by appropriate behavioral patterns. This 
can be evidenced from the results were $18.8 \%$ were found positive for STIs using the syndromic approach and $82 \%$ mentioned having suffered from STIs in the past 6 months more than once. These findings are similar to a study done by Sekirime et $\mathrm{a}^{18}$ which also found that the level of knowledge about STDs and their prevention is not matched by sexual behavioural patterns; most participants still practiced risky sexual behaviour and had contracted STDs.

Among those who were found positive for STIs using the syndromic approach, majority $(76.6 \%)$ followed the appropriate health seeking behavior. This involved using authentic medication prescribed from the hospital or from a local health clinic in the community. This is different from a study done by Elahee et $\mathrm{al}^{17}$ which found $70 \%$ of the women did not seek any treatment for the STIs from any healthcare provider. Most of the respondents $(66.6 \%)$ utilized home remedies to treat the infections and only, $23 \%$ of the respondents consulted specialists or a health center.

The cross sectional study design is prone to validity issues which include information bias, selection bias and confounding ${ }^{19}$. However we minimized these biases by developing our questionnaire based on the WHO simplified tool for diagnosis and treatment of STIs using the syndrome approach, training of the four teams of interviewers, carrying out a pilot testing for its appropriateness before commencement of the whole research project. We also divided the slum into four zones during data collection. Confounding by extraneous variables in the data was minimized by restriction of study participants according to the eligibility criteria.

\section{Conclusion}

Most of the participants knew about types, symptoms, predisposing factors and means of prevention of STIs, although majority did not know about the systemic effects or consequences of STIs to their body and the causative micro organisms.

Majority of the participants didn't follow the appropriate behavior patterns despite being knowledgeable about the various methods of prevention of STIs.

\section{Recommendations}

There is need for further investigation in other slums in Uganda to establish the extent to which the slum populations contribute to the total country wide prevalence of STIs which is still high. This will enable priotization of interventions and probably establish which populations need more attention.

There is need to undestand the barriers to healthy practices despite being knowledgeble about them so as to enable proper interventions for sexual health among young women.

A similar study should be done among men living in slums as they are also vulnerable to STIs.

\section{Acknowledgements}

We are very grateful to MEPI MESAU for the grant Award Number: IR24TW008886 (MESAU-MEPI programmatic Award) from the Forgaty international center given to enable us conduct this study. We are also indebted to the Katanga slum leaders most especially the chairman and the women representative for their collaboration and allowing us to access this community. We are very grateful to our Mentor Dr.Arthur Mpimbaza for the support and guidance he accorded throughout the study.

\section{References}

1. WHO. Global burden. 2008.

2. Rassjo EB, Kambugu F, Tumwesigye MN, Tenywa T, Darj E. Prevalence of sexually transmitted infections among adolescents in Kampala, Uganda, and theoretical models for improving syndromic management. Journal of Adolescent Health. 2006 Mar;38(3):213-21.

3. UBOS. AIDS survey 2011.

4. Hawkes S, Morison L, Chakraborty J, Gausia K, Ahmed F, Islam SS, et al. Reproductive tract infections: prevalence and risk factors in rural Bangladesh. Bulletin World Health Organ. 2002;80(3):180-8.

5. Gottlieb SL, Low N, Newman LM, Bolan G, Kamb $\mathrm{M}$, Broutet N. Toward global prevention of sexually transmitted infections (STIs): the need for STI vaccines. Vacine. 2014 Mar 20;32(14):1527-35.

6. Katusiime C, Schlech WF, 3rd, Parkes-Ratanshi R, Sempa J, Kambugu A. Characteristics of Sexually Transmitted Infections among High-Risk HIV-Positive Patients Attending an Urban Clinic in Uganda. J Int Assoc Provid AIDS Care. 2013 Oct 21.

7. Fontenot HB, George ER. Sexually transmitted infections in pregnancy. Nurs Womens Health. 2014 FebMar;18(1):67-72.

8. Rizwan S, Rath RS, Vivek G, Nitika, Anant G, Farhad A, et al. KAP Study on Sexually Transmitted Infections/Reproductive Tract Infections (STIs/RTIs) among married women in rural Haryana. Indian Dermatol Online J. 2015 Jan-Feb;6(1):9-12. 
9. Seth P, Raiford JL, Robinson LS, Wingood GM, Diclemente RJ. Intimate partner violence and other partner-related factors: correlates of sexually transmissible infections and risky sexual behaviours among young adult African American women. Sex Health. 2010 Mar;7(1):25-30.

10. Seth P, Raiji PT, DiClemente RJ, Wingood GM, Rose E. Psychological distress as a correlate of a biologically confirmed STI, risky sexual practices, self-efficacy and communication with male sex partners in African-American female adolescents. Psychol Health Med. 2009 May;14(3):291-300.

11. Mbonye M, Nalukenge W, Nakamanya S, Nalusiba B, King R, Vandepitte J, et al. Gender inequity in the lives of women involved in sex work in Kampala, Uganda. J Int AIDS Soc. 2012;15 Suppl 1:1-9.

12. WHO. World Health Organization/Regional Office for Africa, HIV/AIDS Epidemiological Surveillance Update for the WHO African Region 2002: Country Profiles. 2003: Harare. 2003
13. WHO.Guidelines for managment of STI. 2003

14. Aggarwal AK, Kaur M, Kumar R et al.Community-based study of reproductive tract infections, including sexually transmitted infections, among the rural population of Punjab, India. Indian Journal of Community Medicine 2009; 34(4): 359-361

15. Uganda Service Provision Assessment Survey. Key findings on HIV/AIDS and STIs.2007

16. UBOS. Uganda Demographics and Health Survey. 2011

17. Elahee SM, Mahmud S, Tanvir S, Zishan R. Breaking the Silence: Reproductive Tract Infections (RTIs) among Women in Slums of Khulna City, Bangladesh. Bangladesh e-Journal of Sociology 2013; 10 (2)

18. Kigongo SW,Tamale J, Lule JC,Wabwire FM. Knowledge, attitude and practice about sexually transmitted diseases among University students in Kampala. Journal of African Health Sciences 2001; 1(1): 16-22.

19. David GK, Lawrence LK, Hal M.Epidemilogical Research;Principles and qualitative Methods. ISBN:9780-471-28985-2. May 1982. 